\title{
A nonlinear bilaplacian equation with hinged boundary conditions and very weak solutions: analysis and numerical solution
}

\author{
Iñigo Arregui · Jesús Ildefonso Díaz · Carlos Vázquez
}

Received: 14 April 2013 / Accepted: 23 September 2013 / Published online: 11 October 2013

(C) Springer-Verlag Italia 2013

\begin{abstract}
We study linear and nonlinear bilaplacian problems with hinged boundary conditions and right hand side in $L^{1}(\Omega: \delta)$, with $\delta=\operatorname{dist}(x, \partial \Omega)$. More precisely, the existence and uniqueness of the very weak solution is obtained and some numerical techniques are proposed for its approximation.
\end{abstract}

Keywords Very weak solutions - Distance to the boundary - Nonlinear bilaplacian operator $\cdot$ Hinged boundary conditions $\cdot$ Numerical methods $\cdot$ Finite elements

Mathematics Subject Classification $\quad 35 \mathrm{G} 50 \cdot 35 \mathrm{G} 60 \cdot 74 \mathrm{G} 25 \cdot 74 \mathrm{G} 15$

\section{Introduction}

Once a not too smooth source datum $f$ is prescribed, the concepts of weak and very weak solutions must be introduced in order to solve the boundary valueproblem

I. Arregui and C. Vázquez have been partially funded by MCINN of Spain (Project MTM2010-21135-C02-01) and Xunta de Galicia (Ayuda CN2011/004 cofunded with FEDER). J. I. Díaz has been partially supported by DGISPI of Spain (Project MTM2011-26119), the Research Group MOMAT (Ref. 910480) supported by UCM and ITN FIRST of the Seventh Framework Program of the European Community's (Grant agreement 238702).

I. Arregui · C. Vázquez $(\bowtie)$

Department of Mathematics, Faculty of Informatics, University of A Coruña, Campus Elviña s/n, 15071 Coruña, Spain

e-mail: carlosv@udc.es

I. Arregui

e-mail: arregui@udc.es

J. I. Díaz

Department of Applied Mathematics, Instituto de Matemática Interdisciplinar, Complutense University of Madrid, Plaza de las Ciencias 3, 28040 Madrid, Spain

e-mail: diaz.racefyn@insde.es 


$$
\left(P_{L}\right) \begin{cases}L u=f(x) & \text { in } \Omega, \\ \text { +boundary conditions } \equiv(B C) & \text { on } \partial \Omega,\end{cases}
$$

where $L$ denotes a linear elliptic differential operator (of order $2 m, m \in \mathbb{N}$ ) in divergence form and $\Omega \subset \mathbb{R}^{N}$.

More precisely, the usual notion of "weak solution" arises from introducing the "energy space" $V \subset H^{m}(\Omega)$ (which denotes the Sobolev space of order $m$, i.e. such that $D^{\alpha} u \in L^{2}(\Omega)$ for any $\alpha \in \mathbb{N}^{N},|\alpha| \leq m$ ). Thus, when $f$ is not necessarily in $V^{\prime}$, a weaker notion of solution can be introduced leading to a correct mathematical treatment. For instance, for $f \in L_{L o c}^{1}(\Omega)$ the notion of "very weak solution" of problem $\left(P_{L}\right)$ can be introduced by integrating $2 m$-times by parts and by merely requiring that $u \in L^{1}(\Omega)$ and that

$$
\int_{\Omega} u(x) L^{*} \zeta(x) d x=\int_{\Omega} f(x) \zeta(x) d x,
$$

for any $\zeta \in W:=\overline{\left\{\zeta \in C^{2 m}(\bar{\Omega}): \zeta \operatorname{satisfies}(B C)\right\}}{ }^{2 m, \infty}(\Omega)$, after assuming

$$
\int_{\Omega}|f(x) \zeta(x)| d x<\infty, \quad \text { for any } \zeta \in W .
$$

In (1.1) $L^{*}$ denotes the adjoint operator of $L$.

Most of the theory on very weak solutions available in the literature deals with second order equations, for which recent results have been obtained when $f \in L^{1}(\Omega: \delta)$, with $\delta=\operatorname{dist}(x, \partial \Omega)$. This idea was originally introduced in [2] by Haïm Brezis in the seventies (see also [4]). More recently, for higher order equations, in [7] some new results proving that the class of $L_{L o c}^{1}(\Omega)$ data for which the existence and uniqueness of a very weak solution can be obtained is, in general, larger than $L^{1}(\Omega: \delta)$, which is actually the optimal class for second order equations. For instance, for the beam equation with Dirichlet boundary conditions it is proved that the optimal class of data is the space $L^{1}\left(\Omega: \delta^{2}\right)$. However, for the simply supported beam the optimal class of data is again $L^{1}(\Omega: \delta)$. The proof of these results is mainly based on the use of the Green function associated to the corresponding boundary value problem.

An important remaining open problem consists of searching solutions (beyond the class of weak solutions) for the case of a nonlinear operator $L$. The two main limitations in the nonlinear setting are: we cannot integrate $2 m$ times by parts and the absence of any kind of Green function associated to the nonlinear problem. In the previous paper [8] the linear and some nonlinear cases with simply supported beams have been considered. The main goal of this paper is to present some new results concerning very weak solutions for the problem associated to a nonlinear bilaplacian operator with hinged boundaries and also to present some numerical examples that illustrate the theoretical results. Notice that although infinite loads do not appear in practice, the idea is to reproduce possible extremely high loads in reality.

We assume a plate is represented by a rectangular open domain $\Omega \subset \mathbb{R}^{N}$, so that the nonlinear problem consists of finding a function $u$, such that:

$$
\left(P_{\varphi}\right) \begin{cases}-\Delta \varphi(-\Delta u)=f & \text { in } \Omega \subset \mathbb{R}^{N}, \\ u=\varphi(-\Delta u)=0, & \text { on } \partial \Omega,\end{cases}
$$

where:

Assumption 1.1 $\varphi: \mathbb{R} \rightarrow \mathbb{R}$ is a continuous strictly increasing function such that $\varphi(0)=0$. 
A classical example corresponds to the linear case $\varphi(s)=E t^{3} s / 12\left(1-v^{2}\right)$, for any $s \in \mathbb{R}$ (with $E, t$ and $v$ positive constants denoting the Young modulus, the thickness and the Poisson coefficient of the plate, respectively), although many other cases arise in the more diverse fields of applications. In particular, some examples appear in different non Hookean materials: cat iron, stone, caoutchouc, many bioelastic materials and most of the composite ones (such as concrete, for example). By using dimensional analysis we can assume any constant arising in the constitutive law of the material equal to one. So, for instance, a case very treated in the literature is $\varphi(s)=|s|^{\alpha-1} s$ for some $\alpha>0$ (notice that $\alpha=1$ reproduces again the linear case).

\section{Mathematical analysis and very weak solutions for the hinged nonlinear bilaplacian problem}

In the case of the problem $\left(P_{\varphi}\right)$, the following notion of very weak solution can be introduced:

Definition 2.1 Given $f \in L^{1}(\Omega: \delta)$, with $\delta=\operatorname{dist}(x, \partial \Omega)$, a function $u \in W_{l o c}^{2,1}(\Omega)$ is a "very weak solution" of $\left(P_{\varphi}\right)$ if $u \in W^{2,1}(\Omega) \cap W_{0}^{1,1}(\Omega), \varphi(-\Delta u) \in L^{1}(\Omega)$ and for any $\zeta \in W^{2, \infty}(\Omega) \cap W_{0}^{1, \infty}(\Omega)$ we have

$$
\int_{\Omega} \varphi(-\Delta u(x))(-\Delta \zeta(x)) d x=\int_{\Omega} f(x) \zeta(x) d x .
$$

We point out that the integral of the right hand side in Definition 2.1 is well justified since it is well-known that any $\zeta \in W_{0}^{1, \infty}(\Omega)$ must satisfy that $|\zeta(x)| \leq \delta(x)\|\nabla \zeta\|_{L^{\infty}(\Omega)}$.

We shall need the following quite weak assumption on the domain $\Omega$ :

Assumption 2.2 There exists the Green function $G_{\Omega}$ (defined at the point $(x, \xi)$ ) for the operator $-\Delta$ with homogeneous Dirichlet boundary conditions on $\partial \Omega$.

It is well known (see, e. g. the books by Stakgold [16] and Friedman [13]) that if, for instance, $\Omega$ is a bounded open set with Lipschitz continuous boundary $\partial \Omega$ then the Green function $G_{\Omega}$ does exist. Moreover, in this case the representation formula (similar to the one given in Theorem 3.1 in [8]) becomes:

$$
u(x)=\int_{\Omega} \varphi^{-1}\left(\int_{\Omega} f(\sigma) G_{\Omega}(s, \sigma) d \sigma\right) G_{\Omega}(x, s) d s \text { for a.e. } x \in \Omega,
$$

once we know the existence (and positivity) of the Green function $G_{\Omega}[13,16]$.

In the proof of the forthcoming main result we use the following lemma.

Lemma 2.3 (Crandall-Tartar [6]) Let $X, Y$ be two vector lattices and $\lambda_{X}, \lambda_{Y}$ be nonnegative linear functionals on $X$ and $Y$, respectively. Let $C \subseteq X$ and $f, g \in C$ imply $f \vee g \in$ $C$. Let $T: C \rightarrow Y$ satisfy $\lambda_{X}(f)=\lambda_{Y}(T(f))$ for $f \in C$. Then $(a) \Rightarrow(b) \Rightarrow(c)$ where (a), (b), (c) are the properties: (a) $f, g \in C$ and $f \leq g$ imply $T(f) \leq T(g),(b)$ $\lambda_{Y}\left((T(f)-T(g))_{+}\right) \leq \lambda_{X}\left((f-g)_{+}\right)$for $f, g \in C,(c) \lambda_{Y}(|T(f)-T(g)|) \leq \lambda_{X}(|f-g|)$. Moreover, if $\lambda_{Y}(F)>0$ for any $F>0$, then $(a),(b),(c)$ are equivalent.

Theorem 2.4 (a.1) Sufficiency. Let us suppose Assumption 1.1 as well as

$$
|r| \leq C_{1}|\varphi(r)|+C_{2} \quad \text { for any } r \in \mathbb{R} .
$$


Then, for any $f \in L^{1}(\Omega: \delta)$ there exists a unique very weak solution of $\left(P_{\varphi}\right)$. Moreover, $u$ is given as $u=D(f)$, with $D: L^{1}(\Omega: \delta) \rightarrow L^{1}(\Omega)$ the nonlocal operator defined by

$$
D(f)=\int_{\Omega} \varphi^{-1}\left(\int_{\Omega} f(\sigma) G_{\Omega}(s, \sigma) d \sigma\right) G_{\Omega}(x, s) d s \text { for a.e. } x \in \Omega,
$$

and if $D(g)=v$ then the following weak maximum principle holds:

$$
f(x) \leq g(x) \text { a.e. in } \Omega
$$

implies that

$$
-\Delta u(x) \leq-\Delta v(x)
$$

and so

$$
u(x) \leq v(x) \text { a.e. } x \in \Omega .
$$

Moreover, if we assume additionally that $\varphi$ is locally Lipschitz continuous, i.e., for any $K>0$ there exists a constant $L(K)>0$ such that

$$
\left|\varphi\left(r_{1}\right)-\varphi\left(r_{2}\right)\right| \leq L(K)\left|r_{1}-r_{2}\right| \text { for any } r_{1}, r_{2} \in[-K, K],
$$

then we have the estimate

$$
\int_{\Omega}[u(x)-v(x)]_{+} d x \leq C(\widehat{K}) \int_{\Omega}\left[\int_{\Omega}[f(\sigma)-g(\sigma)]_{+} G(x, \sigma) d \sigma\right] d x
$$

for some positive constant $C(\widehat{K})$ depending on $\widehat{K}=\max \left\{\|f\|_{L^{1}(\Omega: \delta)},\|g\|_{L^{1}(\Omega: \delta)}\right\}$, where, in general, $h_{+}=\max (0, h)$ and $G(s, \sigma)$ is the Green function for the operator $-\Delta$ with homogeneous Dirichlet boundary conditions on $\Omega$.

Moreover $u$ is smoother than said at Definition 2.1 since, at least, $u \in W_{0}^{1, s}(\Omega: \delta)$ for any $1 \leq s<(N-1)$ and if $f \in L^{1}\left(\Omega: \delta^{\alpha}\right)$ for some $0 \leq \alpha<1$ then $|\nabla \varphi(-\Delta u(x))|$ belongs to the Lorentz space $L^{\frac{N}{N-1+\alpha}}, \infty(\Omega)$.

(a.2) Sufficiency If condition (2.2) is replaced by the additional condition on function $f$

$$
f \in L^{p}(\Omega: \delta) \text { for } p>(N+1) / 2,
$$

then all the conclusions of part (a.1) remain valid for any function $\varphi$ satisfying merely the structural Assumption 1.1.

(b) Strong maximum principle Let $f \in L^{1}(\Omega: \delta)$ with $f \geq 0$ a.e. $x \in \Omega, f \neq 0$. Then the very weak solution satisfies that

$$
\varphi(-\Delta u)(x) \geq C\left(\int_{\Omega}\left[\int_{\Omega} f(\sigma) G_{\Omega}(s, \sigma) d \sigma\right] \delta(s) d s\right) \delta(x)>0,
$$

for a.e. $x \in \Omega$, and

$$
u(x) \geq C\left(\int_{\Omega} \varphi^{-1}\left\{C\left(\int_{\Omega}\left[\int_{\Omega} f(\sigma) G_{\Omega}(s, \sigma) d \sigma\right] \delta(s) d s\right) \delta(y) d y\right\}\right) \delta(x)>0,
$$

for a.e. $x \in \Omega$, and for some positive constant $C$ independent of $f$. 
(c) Necessity Assume that $f \in L_{L o c}^{1}(\Omega)$, such that $f \geq 0$ a.e. in $\Omega$. If $\int_{\Omega} f(x) \delta(x) d x=$ $+\infty$ then it can not exist any very weak solution of $\left(P_{\varphi}\right)$.

Proof Consider the auxiliary problem

$$
\begin{cases}-\Delta m=f & \text { in } \Omega \\ m=0, & \text { on } \partial \Omega .\end{cases}
$$

Since $f \in L^{1}(\Omega: \delta)$, it is well know that

$$
m(x)=\int_{\Omega} f(\sigma) G_{\Omega}(s, \sigma) d \sigma \quad \text { a.e. } x \in \Omega .
$$

Next, we can apply the results in $[10,11]$ to get $m \in L^{N^{\prime}, \infty}(\Omega)\left(\subset L^{N^{\prime}}(\Omega)\right)$, with $N^{\prime}=$ $N /(N-1)$ if $N \geq 2$ and $N^{\prime}=\infty$ if $N=1$. Moreover, from the condition (2.2) we know that if we define $F:=\varphi^{-1}(m)$ then, at least, $F \in L^{N^{\prime}}(\Omega) \subset L^{1}(\Omega: \delta)$, and so operator $D$ given by (2.3) is correctly defined. Now it is a routine matter to check that $u=D(f)$ satisfies the requirements of Definition 2.1, so that $u$ is a very weak solution. In order to prove the uniqueness let $v$ be any very weak solution associated to a given $g \in L^{1}(\Omega: \delta)$, and let $m_{g}=\varphi(-\Delta v)$. Since

$$
\begin{cases}-\Delta\left(m-m_{g}\right)=f-g & \text { in } \Omega, \\ m-m_{g}=0, & \text { on } \partial \Omega,\end{cases}
$$

then we know, again, that

$$
\left(m-m_{g}\right)(x)=\int_{\Omega}(f(\sigma)-g(\sigma)) G_{\Omega}(x, \sigma) d \sigma \quad \text { a.e. } x \in \Omega .
$$

Thus, we have

$$
[(\varphi(-\Delta u)+\varphi(-\Delta v))]_{+}(x)=\left[\int_{\Omega}(f(\sigma)-g(\sigma)) G_{\Omega}(x, \sigma) d \sigma\right]_{+} .
$$

In particular, since $\varphi$ is strictly increasing $f(x) \leq g(x)$ implies that $-\Delta(u-v) \leq 0$ in $\Omega$ and since $u-v=0$ on $\partial \Omega$, we deduce the comparison $u(x) \leq v(x)$ in $\Omega$. Obviously, this implies the uniqueness of the very weak solution.

In order to get the quantitative estimate (2.5) we can adapt the argument already used in [8] for the linear one-dimensional case. Indeed, from the representation formula $u=D(f)$ we get that

$$
\int_{\Omega} u(x) d x=\int_{\Omega} D(f)(x) d x .
$$

So, we can apply the Lemma 2.3. Thus, to prove the $L^{1}$-estimate (2.5) we take $C=X=$ $L^{1}(\Omega: \delta), Y=L^{1}(\Omega), \lambda_{Y}(e)=\int_{\Omega} e(x) d x, T(f)=D(f)$ and

$$
\lambda_{X}(f)=\int_{\Omega}\left(\int_{\Omega} \varphi^{-1}\left(\int_{\Omega} f(\sigma) G_{\Omega}(s, \sigma) d \sigma\right) G_{\Omega}(x, s) d s\right) d x .
$$


Then, thanks to (2.10) and the weak maximum principle we get (b) of Lemma 2.3 which implies that

$$
\int_{\Omega}\left[-m(x)+m_{g}(x)\right]_{+} d x \leq \int_{\Omega}\left[\int_{\Omega}[f(\sigma)-g(\sigma)]_{+} G_{\Omega}(x, \sigma) d \sigma\right] d x,
$$

But we know that $\Delta u(x)=\varphi^{-1}(m(x))$ and $\Delta v(x)=\varphi^{-1}\left(m_{g}(x)\right)$. Then, since $\varphi^{-1}(m(x))$ and $\varphi^{-1}\left(m_{g}(x)\right)$ are in $C(\bar{\Omega})$ the same happens with $u$ and $v$. Taking $K=$ $\max \left\{\|u\|_{L^{\infty}(\Omega)},\|v\|_{L^{\infty}(\Omega)}\right\}$ we can apply the locally Lipschitz assumption on $\varphi$ to conclude that

$$
\int_{\Omega}[-\Delta u(x)+\Delta v(x)]_{+} d x \leq L(K) \int_{\Omega}\left[\int_{\Omega}[f(\sigma)-g(\sigma)]_{+} G_{\Omega}(x, \sigma) d \sigma\right] d x .
$$

Finally, applying the same arguments than before but now for $u$ and $v$ instead $m$ and $m_{g}$ we get the estimate $(2.5)$ for some positive constant $C(\widehat{K})$ depending on $\widehat{K}=$ $\max \left\{\|f\|_{L^{1}(\Omega: \delta)},\|g\|_{L^{1}(\Omega: \delta)}\right\}$.

The additional regularity of part (a.1) is a direct application of the results in [10]. The proof of part (a.2) is similar once that we recall that the condition (2.6) implies that $m \in$ $L^{\infty}(\Omega)$ (see e.g. Proposition 2.1 of [15]). Then, for any function $\varphi$ satisfying Assumption 1.1 we can define the function $F:=\varphi^{-1}(m)$ and we get that $F \in L^{\infty}(\Omega)$, so that we can define again $u$ as the solution of

$$
\begin{cases}-\Delta u=F & \text { in } \Omega \\ u=0, & \text { on } \partial \Omega\end{cases}
$$

and the rest follows as in the proof of part (a.1).

The proof of the strong maximum principle uses the following estimate: if

$$
\begin{cases}-\Delta U=F & \text { in } \Omega \\ U=0, & \text { on } \partial \Omega\end{cases}
$$

with $F \in L^{1}(\Omega: \delta)$ and $F \geq 0$, then there exists a positive constant $C$ such that

$$
U(x) \geq C\left(\int_{\Omega} F(s) \delta(s) d s\right) \delta(x)>0 \quad \text { a.e. } x \in \Omega .
$$

This result was first proved first by Morel and Oswald (in an unpublished manuscript by 1985) and later developed in [3]. Thus, applying it to function $m$ we get (2.7) and applying it again, now to (2.11), we conclude estimate (2.8).

In order to prove part (c), and more specifically the complete blow up (in the whole domain $\Omega$ ) when $f \notin L^{1}(\Omega: \delta)$, we truncate $f$ generating the sequence $f_{n}(x)=\min (f(x), n)$. Now, if $u_{n}$ is the associated solution (notice that $f_{n} \in L^{\infty}(\Omega) \subset L^{1}(\Omega: \delta)$ ), then $u_{n}(x) \geq$ $\alpha\left(\left\|f_{n}\right\|_{L^{1}(\Omega: \delta)}\right) \delta(x)$, for a suitable increasing function $\alpha$ such that $\alpha\left(\left\|f_{n}\right\|_{L^{1}(\Omega: \delta)}\right) \nearrow+\infty$ as $n \nearrow+\infty$, which implies that $u_{n}(x) \nearrow+\infty$ a.e. $x \in \Omega$. The proof is now completed.

Remark 2.5 It seems possible to replace assumption (2.6), in part (a.2), by some other additional information on $f$ of a different nature like

$$
0 \leq f(x) \leq \delta(x)^{-\beta} \text { for some } \beta<2, \text { a.e. } x \in \Omega .
$$


Indeed, arguing as in [9] it can be shown that $0 \leq m(x) \leq \delta(x)^{\theta}$ for some $\theta>0$, a.e. $x \in \Omega$, and thus a growth assumption on $\varphi$ of the type

$$
|r|^{\omega} \leq C_{1}|\varphi(r)|+C_{2} \text { for any } r \in \mathbb{R}, \quad \text { and for some } \omega>1,
$$

could imply that $F=\varphi^{-1}(m) \in L^{1}(\Omega: \delta)$ which in turn implies the correct definition of operator $D$ given by (2.3). The details will be given elsewhere.

Remark 2.6 The above Theorem 2.4 can be suitably applied to get the existence of very weak solutions of singular perturbed problems of the type

$$
\begin{cases}-\Delta \varphi(-\Delta u)=\frac{h}{u^{a}} & \text { in } \Omega, \\ u=\varphi(-\Delta u)=0 & \text { on } \partial \Omega,\end{cases}
$$

with $h=\frac{g}{\delta^{b}}, g \in L^{\infty}(\Omega)$ such that $0<C_{g} \leq g(x)$ for some $a, b \geq 0$. This fact was mentioned in the one-dimensional case in [8] although the proof is exactly the same for the n-dimensional case. For some results on a singular perturbation problem, although for the case of Dirichlet boundary conditions, see [14].

Remark 2.7 As in Corollary 4.2 in [8], it is possible to get a rigorous proof of the convergence, at least in $W_{0}^{1, s}(\Omega: \delta)$ for any $1 \leq s<(N-1)$, of the solutions $u_{\varepsilon}$ associated to a sequence of data $f_{\varepsilon}$ such that $f_{\varepsilon} \rightarrow f$ in $L^{1}(\Omega: \delta)$.

\section{Numerical methods}

In order to illustrate the theoretical results of previous sections, we have considered the numerical solution of different examples of linear and nonlinear problems. The starting point is to follow the steps of the theoretical proof, so that we decompose problem $\left(P_{\varphi}\right)$ into two recursive second order problems with homogeneous Dirichlet boundary conditions:

$$
\begin{gathered}
\left(P_{\varphi}^{1}\right) \begin{cases}-\Delta m=f & \text { in } \Omega, \\
m=0, & \text { on } \partial \Omega,\end{cases} \\
\left(P_{\varphi}^{2}\right) \begin{cases}-\Delta u=\varphi^{-1}(m) & \text { in } \Omega, \\
u=0, & \text { on } \partial \Omega .\end{cases}
\end{gathered}
$$

Note that the previous decomposition has already been used in the frame of elastohydrodynamic lubrication problems in which the surface deformation is governed by a plate equation $[1,12]$ for a more regular right hand side. For the choices of $f \in L^{1}(\Omega: \delta)$ to be considered in the forthcoming numerical examples, we approximate them by a convenient sequence $\left\{f_{\epsilon}\right\}_{\epsilon} \subset L^{2}(\Omega), f_{\epsilon} \rightarrow f$ in $L^{1}(\Omega: \delta)$. In practice, we show the numerical results for $\epsilon$ small enough.

For the numerical discretization of problems $\left(P_{\varphi}^{1}\right)$ and $\left(P_{\varphi}^{2}\right)$, we use piecewise linear Lagrange finite elements. Note that in [5] for second order elliptic problems with $L^{\infty}(\Omega)$ coefficients and $L^{1}(\Omega)$ right hand side, the convergence of the numerical method to the unique "renormalized" solution has been obtained.

Additionally, in order to better capture the solution near the region with steepest gradients, in the present paper we apply an adaptive refinement based on the computed gradients of the solution. For this purpose, we propose an adaptive remeshing algorithm that uses the gradient of the solution as the metric for the refinement procedure. Thus, after computing the gradient 
of approximated solution which is constant for each element as we are using piecewise linear finite element, we just refine the elements that exhibit larger gradients. Once the elements to be refined have been identified according to the previous criterium, each triangle is divided into four subtriangles, the mid-point of the longest side being connected with the opposite vertex and the other two sides mid-points. Next, in order to ensure the conformity of the new mesh, an additional refining step (by subdivision into two or three subtriangles) has to be performed.

In practice, for the computation of the integrals we have employed numerical quadrature formulae over the triangles, the quadrature nodes being the vertices for all the examples.

\section{Numerical examples}

Among all numerical examples that have been carried out, in the present section the more illustrative ones are shown. In all tests we take $\Omega=(0,1) \times(0,1)$. Except for Test 4 , we approximate $f=f_{0}$ in the form:

$$
f_{\epsilon}(x, y)=\frac{1}{(x+\epsilon)^{k}} \frac{1}{(1+\epsilon-x)^{k}} \frac{1}{(y+\epsilon)^{k}} \frac{1}{(1+\epsilon-y)^{k}},
$$

with $\epsilon=10^{-14}$. We note that "formally" $f$ behaves as $\delta^{-k}$ so that $f \in L^{1}\left(\Omega: \delta^{\alpha}\right)$ for some $\alpha \in[0,1]$ if and only if $k<1+\alpha$. For instance, if $k=2$ then $f \notin L^{1}(\Omega: \delta)$ while if $k=1$ then $f \in L^{1}\left(\Omega: \delta^{\alpha}\right)$ for any $\alpha \in(0,1]$. As a matter of fact for $k=1 f \in L^{p}(\Omega: \delta)$ for $p \in[1,2)$ and thus the solution $m$ of problem $\left(P_{\varphi}^{1}\right)$ satisfies that $m \in L^{\infty}(\Omega)$, since in this case $\Omega \subset \mathbb{R}^{2}$. This explains that the problem $\left(P_{\varphi}\right)$ is well-posed for any $\varphi$ satisfying Assumption 1.1 (see the part (a) of Theorem 2.4).

Moreover, for $k=1$ we know that $\|\nabla m\| \in L^{\frac{2}{1-\alpha}, \infty}(\Omega)$. Notice that, "roughly speaking", if $\|\nabla m\|$ behaves as $\delta^{-\beta}$ near $\partial \Omega$ then the above integrability requires that $2 \beta<1$. Also we notice that for $\epsilon=0$ Tests 1 to 3 correspond to the choice $k=1$ that guarantees $f \in L^{1}(\Omega: \delta)$ and $f \notin L^{1}(\Omega)$, while Test 5 corresponds to $k=2$, in which $f \notin L^{1}(\Omega: \delta)$.

Notice that although in the literature there are available many equivalent expressions for the Green function $G_{\Omega}$ (mentioned in Theorem 2.4), we shall not use any one of them in our numerical methods.

\subsection{Test 1: a first linear problem}

We first consider a linear case, that corresponds to $\varphi(s)=s$ and $k=1$ in (4.1). Figure 1 shows the computed values of $u_{h}$ and $m_{h}=\varphi\left(-\Delta u_{h}\right)$ on a uniform triangular mesh with 16,641 vertices and 32,768 elements. Figure 2 shows an adaptive mesh (with 7,325 nodes and 14,136 elements) and the computed values of $m_{h}$. Next, Fig. 3 shows the product of different powers of the distance multiplied by the norm of the gradient of $m_{h}$ with adaptive refinement. This figure particularly illustrates how the gradient of $m_{h}$ blows up at the boundary and tends to zero when multiplied by the different increasing powers of the distance. More precisely, for $p=0$ we observe that $\delta^{p}\left\|\nabla m_{h}\right\|$ becomes unbounded near $\partial \Omega$ as soon as we refine the mesh, although if we take $p=1$ then $\delta^{p}\left\|\nabla m_{h}\right\|$ remains bounded near $\partial \Omega$. This last comment is illustrated by Table 1 , where the evolution of the maximum of $\delta^{p}\left\|\nabla m_{h}\right\|$ is shown. 

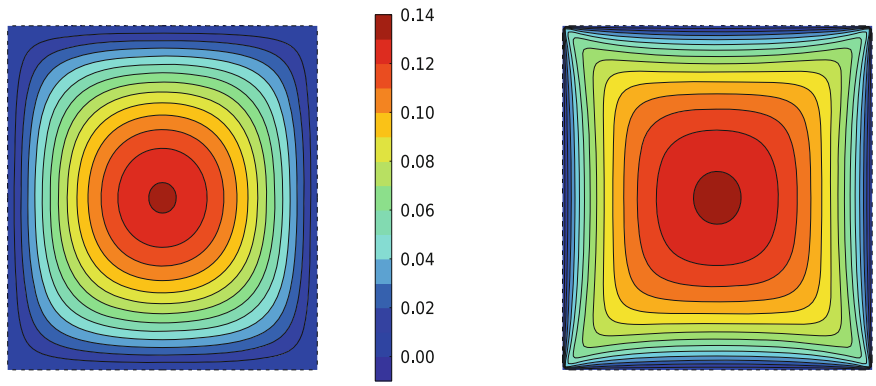

2.1

1.8

1.5

1.2

0.9

0.6

0.3

0.0

Fig. 1 Numerical solutions $u_{h}$ (left) and $m_{h}=-\Delta u_{h}$ (right) in Test 1
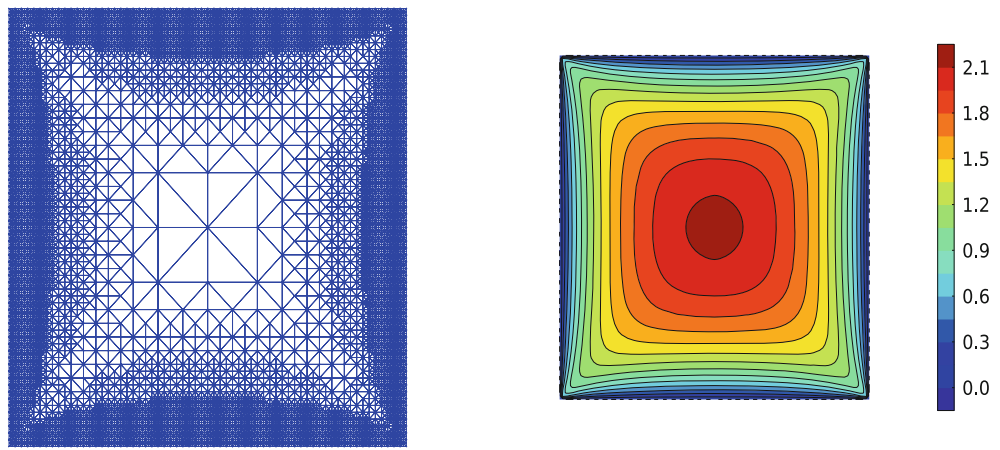

Fig. 2 Adaptive mesh and computed $m_{h}=-\Delta u_{h}$ in Test 1, after seven refinement steps
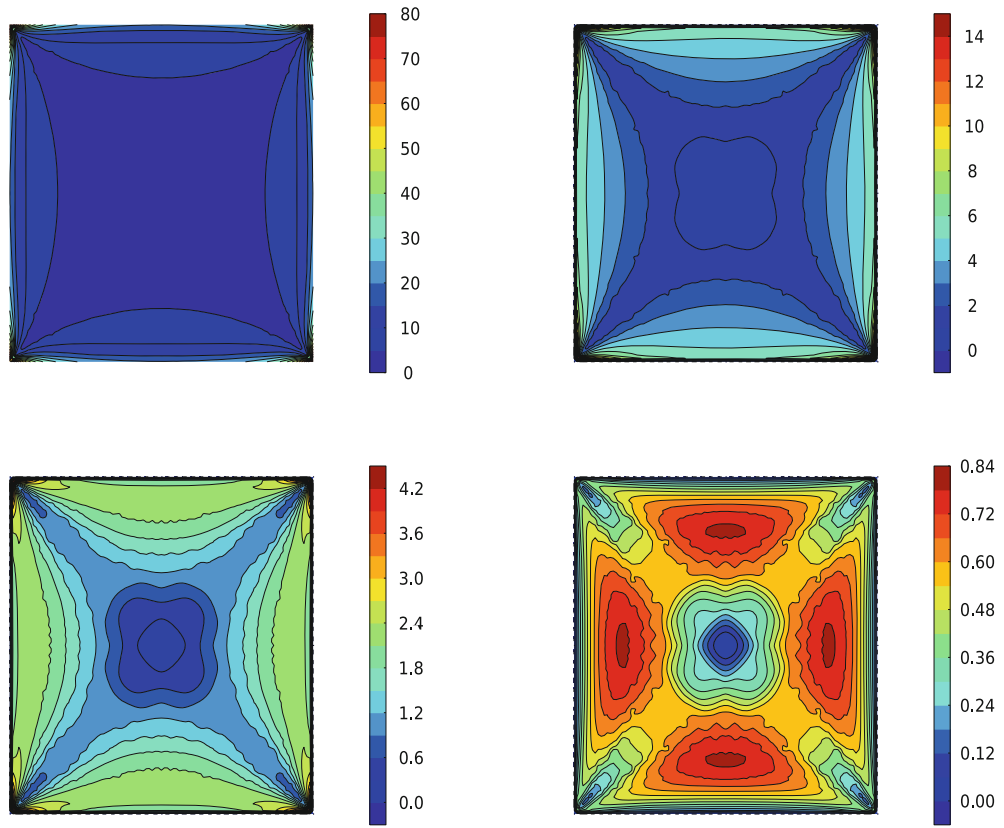

Fig. 3 Computed isolines of the function $\left\|\delta^{p} \nabla m_{h}\right\|$, for $p=0,0.5,1,1.5$ (from left to right and from top to bottom) for Test 1 
Table 1 Evolution of the maximum of $\delta^{p}\left\|\nabla m_{h}\right\|$ with 5-7 refinement steps for different values of $p$

\begin{tabular}{llrr}
\hline$p$ & \multicolumn{3}{l}{ Number of vertices } \\
\cline { 2 - 4 } & 925 & 2,817 & \multicolumn{1}{c}{7,325} \\
\hline 0 & 21.6082 & 40.8855 & 79.5934 \\
0.25 & 6.1135 & 8.9210 & 14.1330 \\
0.50 & 2.5704 & 3.1541 & 4.2018 \\
0.75 & 1.2717 & 1.2805 & 1.2850 \\
1.00 & 0.7808 & 0.7887 & 0.7916
\end{tabular}
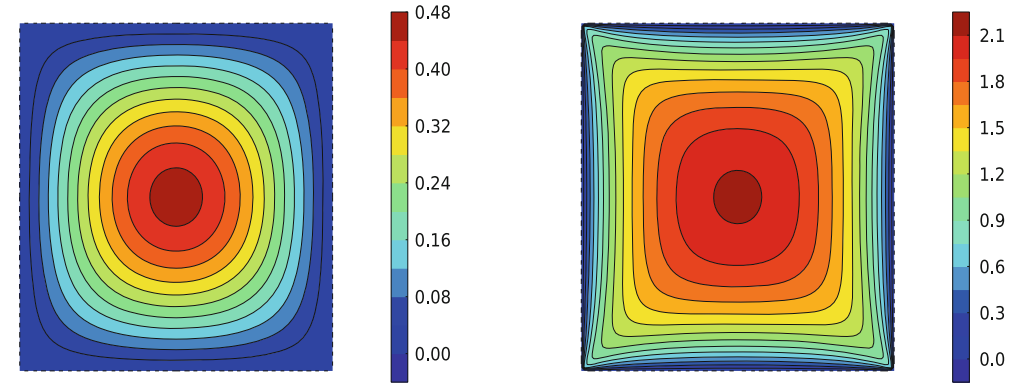

Fig. 4 Numerical solutions $u_{h}$ (left) and $m_{h}=\varphi\left(-\Delta u_{h}\right)$ (right) in Test 2 for the uniform mesh
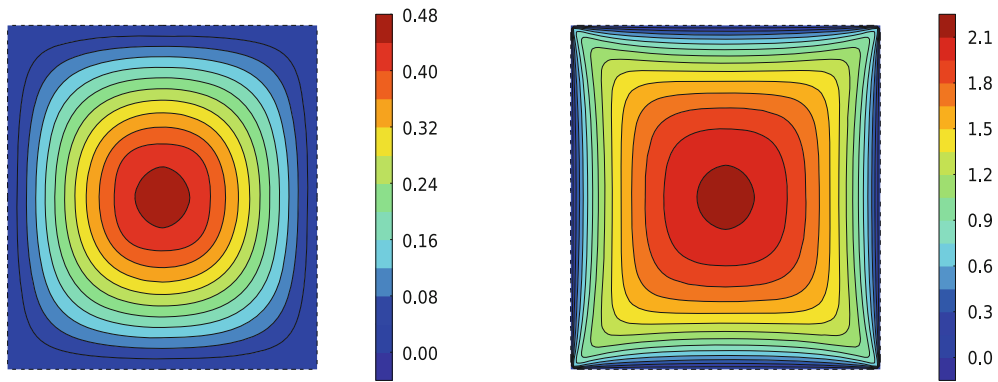

Fig. 5 Numerical solutions $u_{h}$ (left) and $m_{h}=\varphi\left(-\Delta u_{h}\right)$ (right) in Test 2, after seven adaptive refinement steps

4.2 Test 2: a nonlinear problem with $\varphi(s)=s^{1 / 3}$

In this example we consider the nonlinear bilaplacian problem associated to $\varphi(s)=s^{1 / 3}$ and the same choice as in Test 1 for the remaining data. Figures 4 and 5 show the analogous results to Figures 1 and 2, respectively. In this case we notice that the convergence of the solution as $\epsilon \downarrow 0$ is ensured because $\varphi^{-1}$ is a Lipschitz function (see part (a) of Theorem 2.4). 

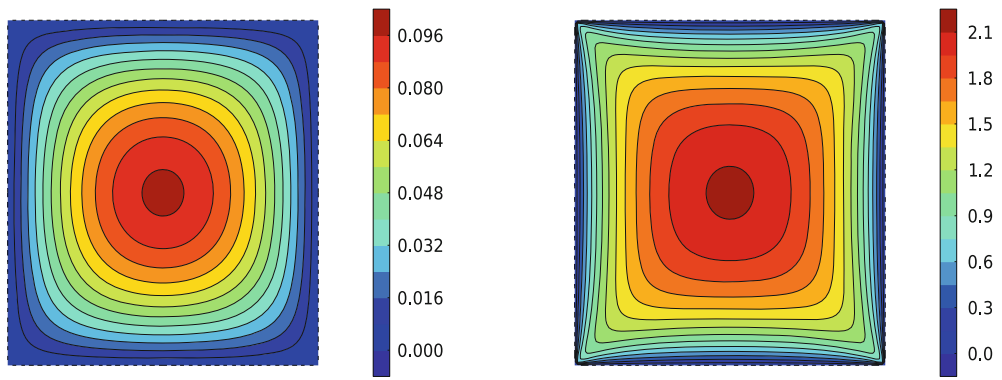

Fig. 6 Numerical solutions $u_{h}(l e f t)$ and $m_{h} \varphi\left(-\Delta u_{h}\right)($ right $)$ in Test 3 for the uniform mesh
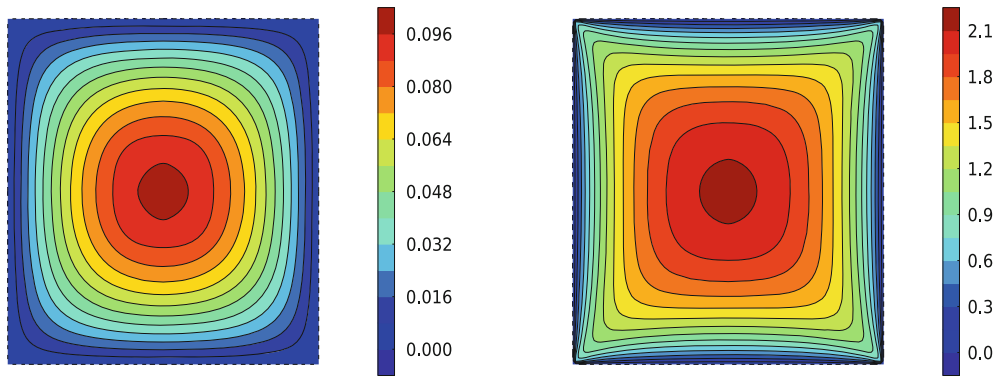

Fig. 7 Numerical solutions $u_{h}$ (left) and $m_{h}=\varphi\left(-\Delta u_{h}\right)$ (right) in Test 3, after seven adaptive refinement steps

\subsection{Test 3: a nonlinear problem with $\varphi(s)=s^{2}$}

In this example we consider the nonlinear bilaplacian problem associated to $\varphi(s)=s^{2}$ and the same choice as in Test 1 for the remaining data. Figures 6 and 7 show the analogous results to Figs. 1 and 2, respectively. In particular, as in Test 1, they illustrate how the saving of computational cost with adaptive refinement preserves the accuracy obtained by uniform refinement as expected. Notice that although $\varphi^{-1}$ is not Lipschitz [and so assumption (2.12) fails], as indicated before, function $f_{\epsilon}$ satisfies condition (2.6) and so the convergence to a very weak solutions is well justified.

\subsection{Test 4: another linear problem with different $f_{\epsilon}$}

As in Test 1 , here we also consider $\varphi(s)=s$ although the right hand side function is now given by:

$$
f_{\epsilon}(x, y)=\frac{1}{(x+\epsilon)} \frac{1}{(1+\epsilon-y)},
$$

with $\epsilon=10^{-14}$. Figure 8 shows the results in case of adaptive refinement, more reasonable than uniform refinement due to the lack of symmetry of the solution. After seven refinement steps the mesh contains 4247 nodes and 8164 elements. In this case $\left\|\nabla m_{h}\right\|$ only blows up near the part of the boundary $\Gamma=\partial \Omega \cap(\{x=0\} \cup\{y=1\})$. Again, if $\delta_{\Gamma}$ denotes the distance to $\Gamma$ function then we have observed that $\delta_{\Gamma}\left\|\nabla m_{h}\right\|$ does not blow up. 

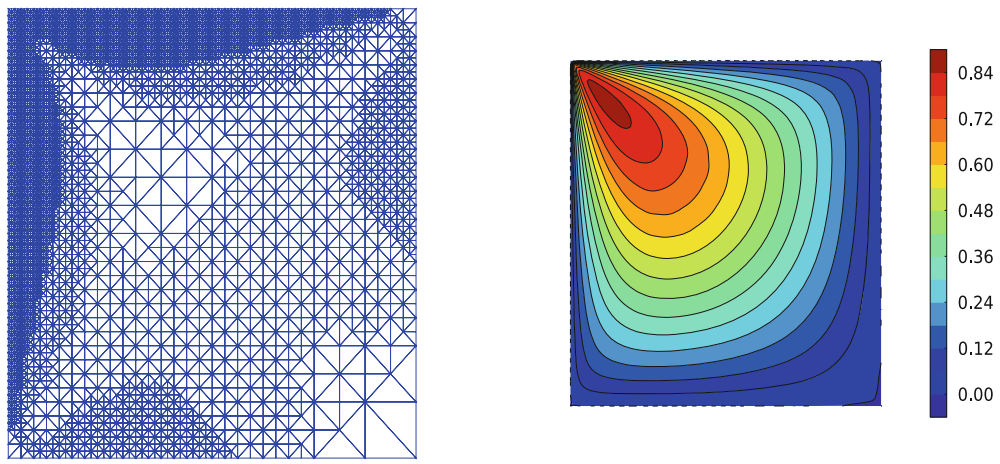

Fig. 8 Adaptive mesh and numerical solution $m_{h}$ in Test 4, after seven refinement steps
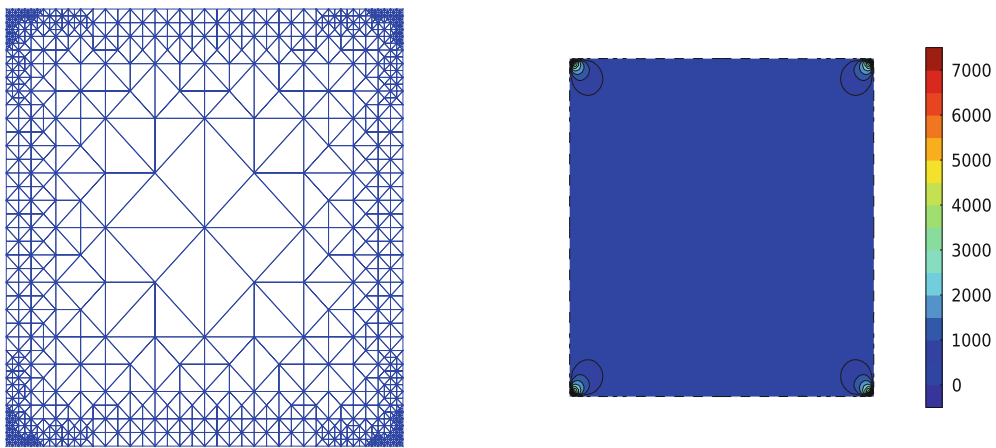

Fig. 9 Adaptive mesh and numerical solution $m_{h}=-\Delta u_{h}$ in Test 5, after seven refinement steps

\subsection{Test 5: a linear problem with $f \notin L^{1}(\Omega: \delta)$}

In this example, we illustrate a case where $f \notin L^{1}(\Omega: \delta)$. For this purpose we choose $k=2$ in (4.1). The remaining data are the same as in Test 1. In this case, we just represent in Fig. 9 the adaptive mesh (containing 993 vertices and 1,760 elements) and the computed value of $m_{h}=-\Delta u_{h}$ to observe the blows up of its gradient near the boundary, in accordance with the theoretical result stating that very weak solutions do not exist (see part (c) of Theorem 2.4).

\section{References}

1. Bayada, G., Durany, J., Vázquez, C.: Existence of a solution for a lubrication problem in elastic journalbearing devices with thin bearing. Math. Methods Appl. Sci. 18, 255-266 (1995)

2. Brezis, H.: Une équation Semi-linéaire Avec Conditions Aux Limites Dans $L^{1}$. Personal communication to J.I. Díaz (unpublished)

3. Brezis, H., Cabré, X.: Some simple nonlinear PDE's without solutions. Bull. UMI 1, 223-262 (1998)

4. Brezis, H., Cazenave, T., Martel, Y., Ramiandrisoa, A.: Blow up for $u_{t}-\Delta u=g(u)$ revisited. Adv. Differ. Equ. 1, 73-90 (1996)

5. Casado-Díaz, J., Chacón-Rebollo, T., Girault, V., Gómez-Mármol, M., Murat, F.: Finite elements approximation of second order linear elliptic equations in divergence form with right-hand side in $L^{1}$. Numer. Math. 105, 337-374 (2007) 
6. Crandall, M.G., Tartar, L.: Some relations between nonexpansive and order preserving maps. Proc. AMS 78(3), 385-390 (1980)

7. Díaz, J.I.: On the very weak solvability of the beam equation. Rev. R. Acad. Cien. Ser. A (RACSAM) 105, 167-172 (2011)

8. Díaz, J.I.: Non Hookean Beams and Plates: Very Weak Solutions and Their Numerical Analysis (2013). (submitted)

9. Díaz, J.I., Hernández, J., Rakotoson, J.M.: On very weak positive solutions to some semilinear elliptic problems with simultaneous singular nonlinear and spatial dependence terms. Milan J. Math. 79, 233-245 (2011)

10. Díaz, J.I., Rakotoson, J.M.: On the differentiability of very weak solutions with right hand side data integrable with respect to the distance to the boundary. J. Funct. Anal. 257, 807-831 (2009)

11. Díaz, J.I., Rakotoson, J.M.: On very weak solutions of semi-linear elliptic equations in the framework of weighted spaces with respect to the distance to the boundary. Discret. Contin. Dyn. Syst. 27, 1037-1058 (2010)

12. Durany, J., García, G., Vázquez, C.: An elastohydrodynamic coupled problem between a piezoviscous Reynolds equation and a hinged plate model. RAIRO Modél. Math. Anal. Numér. 31, 495-516 (1997)

13. Friedman, A.: Generalized Functions and Partial Differential Equations. Prentice-Hall, Englewood Cliffs (1963)

14. Ghergu, M.: A biharmonic equation with singular nonlinearity. Proc. Edinb. Math. Soc. 55, 155-166 (2012)

15. Souplet, $\mathrm{Ph} .:$ A survey on $L_{\delta}^{p}$ spaces and their applications to nonlinear elliptic and parabolic problems. Nonlinear partial differential equations and their applications. GAKUTO Int. Ser. Math. Sci. Appl. 20, 464-479 (2004)

16. Stakgold, I.: Green's functions and boundary value problems. In: Pure and Applied Mathematics Series. Wiley, New York (1998) 